FRERE Séverine, RICHER Cyprien

RTS n 92 (2006) pp. 245-261

\title{
Structuration intercommunale et rôle des politiques \\ de transports publics dans le Valenciennois.
}

\section{Introduction}

L'intercommunalité à fiscalité propre connaît une embellie remarquable depuis la loi Chevènement ${ }^{1}$. Les multiples actes de foi en sa faveur (Vanier, 2004) confèrent aux EPCI de nouveaux pouvoirs en matière de développement économique, d'aménagement, d'habitat, ou de transport, si bien que la coopération intercommunale semble devenir «le paradigme du développement territorial» (Chaboche, 2001). Le renforcement de la cohérence territoriale dans le cadre d'une intercommunalité de projet est placé au cœur des enjeux des évolutions législatives. Plus spécifiquement, c'est l'intégration de compétences sectorielles, et notamment le décloisonnement des politiques de transport public, qui est visée. Seulement, le succès quantitatif de l'intercommunalité ${ }^{2}$ cache difficilement un développement laborieux et des effets insaisissables qui n'entrent pas toujours en résonance avec les grandes espérances ${ }^{3}$.

Cet article présente les interactions entre construction intercommunale et la dynamique des transports publics dans l'arrondissement de Valenciennes. Ce travail est issu d'une étude de $\operatorname{cas}^{4}$ réalisée dans le cadre d'une recherche pour le PREDIT ${ }^{5}$. L'exemple du Valenciennois interroge cette construction intercommunale à travers la difficile émergence d'une institution d'agglomération et à travers ses effets sur la politique des transports publics.

L'arrondissement de Valenciennes compte 350000 habitants répartis dans 82 communes avec une ville centre de petite taille (42 000 habitants). Par l'intermédiaire d'une approche diachronique, nous analyserons l'influence de la recomposition intercommunale du Valenciennois sur le fonctionnement de l'agglomération et l'intégration de ses politiques notamment dans les champs des transports et de l'aménagement. Le développement de l'intercommunalité dans l'arrondissement de Valenciennes favorise-t-il l'émergence de dynamiques internes au territoire? Quel rôle et quelle influence les transports publics exercent-ils dans le développement de ces solidarités émergentes ? Peut-on considérer comme D. Gaxie que l'exemple valenciennois fournit la preuve que «le renforcement de l'intégration [est] le produit d'une histoire cumulative » (Gaxie, 1997) ?

\footnotetext{
${ }^{1}$ Loi relative au renforcement et à la simplification intercommunale dite loi Chevènement, 1999.

${ }^{2} 600$ nouveaux groupements ont été créés en 2005, ce qui porte à 2572 le nombre d'EPCI (80\% de la population française). Par ailleurs, les communautés d'agglomération représentent $42 \%$ des autorités organisatrices de transport urbain en 2003.

${ }^{3}$ Voir Le Saout R., Madoré F (dir), 2004.

${ }^{4}$ Ces études de cas ont été réalisées en 2003 et 2004 dans les régions urbaines de Rennes, Saint-Étienne, Valenciennes, Caen et Saint-Brieuc, à l'occasion d'une recherche financée par l'Ademe, dans le cadre du groupe opérationnel $11 \mathrm{du} 3$ è programme d'innovation et de recherche dans les transports (Prédit). Ces travaux ont associé des chercheurs et doctorants de l'Inrets (S. Frère, C. Gallez, P. Menerault et C. Richer), de l'Université de Paris 12 (C. Guerrinha) et de l'Université de Lille 1 (F. Bodin).

${ }^{5}$ Gallez, Menerault (dir), 2005.
} 
Notre démarche mettra en perspective les dynamiques locales observées dans le Valenciennois avec le contexte national. Cette approche permet de distinguer ce qui relève des singularités locales de ce qui procède d'un mouvement national lié aux différentes évolutions législatives.

L'émergence et la transformation des solidarités intercommunales dans cet arrondissement se décomposent en trois périodes que nous présenterons successivement. La première précède l'intercommunalité de projet et examine les origines de la coopération intercommunale dans l'arrondissement de Valenciennes. Le second temps s'étend de la mise en place de la loi $\mathrm{ATR}^{6}$ à la veille de l'adoption de la loi Chevènement (1999) et présente la difficile structuration intercommunale du Valenciennois dans un contexte où les transports publics demeurent cloisonnés. Enfin, la dernière période, qui débute au lendemain de la mise en œuvre de la Loi Chevènement, analyse la récente tectonique intercommunale et ses effets sur la politique des transports urbains dans l'arrondissement de Valenciennes.

Ainsi, nous montrerons dans un premier temps, qu'à la veille de l'adoption de la loi ATR, l'espace de gestion des transports urbains, presque équivalent à celui de l'arrondissement, apparaît comme le référentiel fonctionnel dans le Valenciennois pour la planification et les projets d'aménagement. Puis, dans un deuxième temps, nous verrons que la fragmentation intercommunale très importante dans les années 1990 constitue un handicap pour le développement d'un projet de Transport Collectif en Site Propre (TCSP) à l'échelle de l'agglomération. Enfin, la troisième partie illustrera les effets controversés des leaderships politiques dans la recherche d'une cohérence spatiale et intersectorielle préconisée par la loi Chevènement.

\footnotetext{
${ }^{6}$ Loi relative à l'Administration Territoriale de la République dite loi Joxe, 1992.
} 


\section{Des années 1960 à la loi ATR (1992) : L'apprentissage de l'intercommunalité et l'émergence de structures de coopération de forme syndicale}

Cette première période, marquée par l'action des ingénieurs de l'État à l'échelle de l'arrondissement, constitue une phase d'apprentissage de l'intercommunalité pour les élus locaux. Elle se prolonge dans les années 1970 avec l'apparition des premières structures de coopération de forme syndicale, alors que le rôle de la DDE va décliner progressivement.

\subsection{Une solidarité territoriale à l'échelle de l'arrondissement formée sous l'impulsion des ingénieurs de l'État.}

Dans les années 1960, la Chambre de Commerce et d'Industrie de Valenciennes impulse la plupart des actions de développement et de modernisation économique. Elle sera à l'origine d'un long processus de mise en synergie des acteurs. Dans ces années, les élus locaux sont restés en retrait des stratégies globales de développement local, qui sont, pour ainsi dire, aux mains des Houillères: "en échange de la sécurité de l'emploi, les élus et la population avaient renoncé à exercer la plus petite influence sur le devenir collectif de l'arrondissement $\gg($ Subra, 1996).

En 1967, la loi d'orientation foncière confie aux services locaux de l'équipement l'élaboration de schémas directeurs d'aménagement et d'urbanisme (SDAU). C'est dans ce cadre que l'équipe constituée autour d'André Talmant, ingénieur d'arrondissement, saura nouer avec les différents responsables locaux, en particulier avec les élus, des relations de collaboration. Cet ingénieur, dont l'éthique de l'action publique consiste à mettre au centre les habitants et leurs représentants, les élus, met en application ces principes à l'occasion de l'élaboration du SDAU de l'arrondissement de Valenciennes. Cette expérience particulièrement novatrice constitue, pour le Valenciennois, la première réflexion globale et «la première occasion qu'ont les élus de travailler ensemble, le premier exemple d'intercommunalité. À un niveau géographique, l'arrondissement, qui semble pertinent parce qu'il recouvre indéniablement une réalité économique et sociale et une identité collective ${ }^{7}$.

Après le départ des deux ingénieurs d'arrondissement André Talmant et Pierre Calame qui lui a succédé, l'implication de la DDE dans l'arrondissement ne cesse de décliner. Malgré la démarche particulièrement originale du premier schéma directeur au début des années 1970, et le foisonnement des études menées sur le Valenciennois dans les années 1980, l'expertise accumulée a finalement peu servi la mise en place de réflexions transversales. La perte de connaissances s'expliquerait donc par l'absence d'un relais technique suffisant à l'échelle locale lorsque les chargés d'études du Groupement d'Etudes et de Programmation quittent la DDE au début des années 1980. Cette période est aussi marquée par les lois de décentralisation qui "achèvent en quelques années le travail de démantèlement de l'outil $D D E »$ dans le Valenciennois (Subra, 1993).

Dans cette période, la première structure de coopération intercommunale, le syndicat intercommunal pour la promotion de l'enseignement supérieur de l'arrondissement de Valenciennes, est créée en 1967 à l'échelle de l'arrondissement en vue d'acquérir les terrains pour l'implantation de l'université. Comme il regroupe les 82 communes de l'arrondissement,

\footnotetext{
${ }^{7}$ Entretien avec P. Subra, 8 décembre 2003.
} 
ce syndicat a acquis parmi ses compétences la révision du Schéma directeur. Il pilote ainsi le second SDAU adopté en 1992 ainsi que la révision du schéma directeur, en 2002. Le syndicat participe ainsi au maintien d'une certaine continuité dans la planification à l'échelle de l'arrondissement.

Cette expérience soulève néanmoins un paradoxe : d'un côté, l'échelle de l'arrondissement semble perdurer comme un niveau géographique de référence, en particulier pour l'élaboration des documents de planification, de l'autre, les structures de coopération semblent de moins en moins en mesure de maintenir les solidarités territoriales entre les 82 communes de l'arrondissement.

\subsection{L’émergence de la coopération intercommunale de forme syndicale}

Parallèlement à cet apprentissage de l'intercommunalité impulsé par la DDE - qui, malgré les efforts de A.Talmant ne sera véritablement l'affaire que de quelques élus locaux- les formes de la coopération intercommunale dans l'arrondissement trahissent la modestie de l'ambition communautaire (figure 1).

Figure 1 : Les SIVOM, premières structures de coopération intercommunale dans le Valenciennois

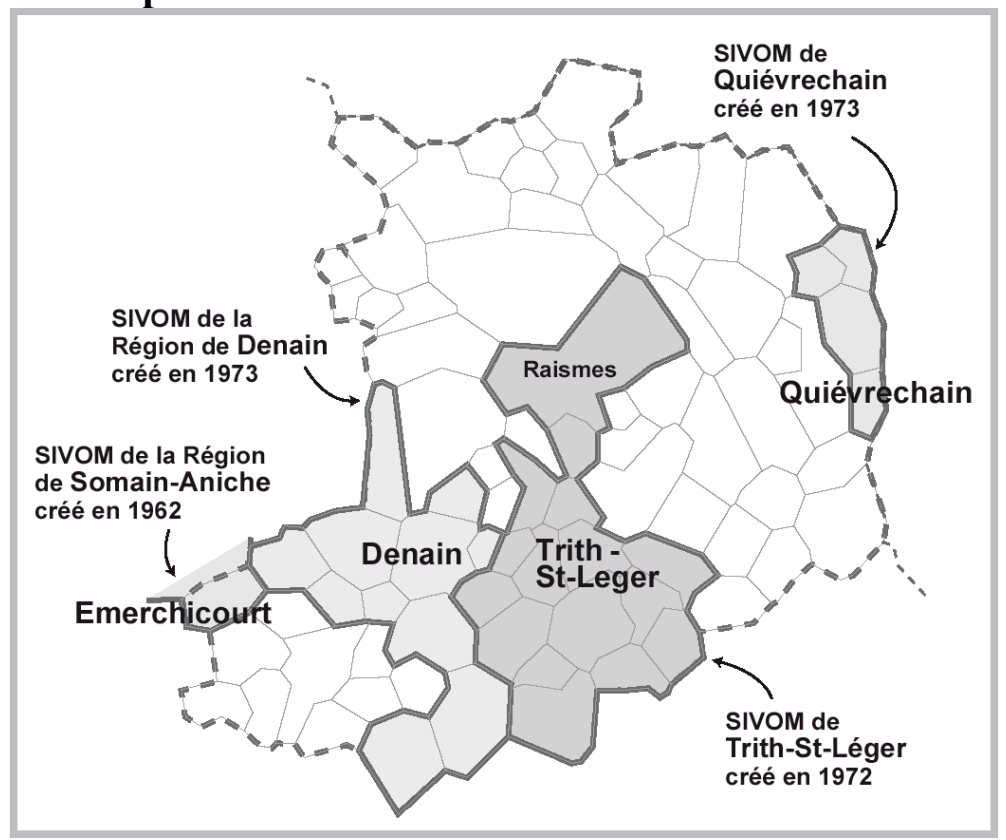

Les deux structures les plus importantes, créées dans les années 1970, prennent la forme de syndicats intercommunaux à vocation multiple (SIVOM). En 1972, se crée le SIVOM de Trith-St-Léger qui regroupe 16 communes. Outre sa compétence économique, il gère également les équipements et services sanitaires et sociaux ainsi que l'eau et la collecte des déchets. En 1973, se crée le SIVOM de Quiévrechain (4 communes) et celui du canton de Denain qui regroupe 11 communes dont les compétences portent sur l'urbanisme, les Zones d'Aménagement Concerté, les équipements culturels, les espaces verts. Ces regroupements s'effectuent surtout selon des critères économiques et fournissent une réponse aux besoins d'équipements communaux.

La coopération intercommunale est un révélateur des difficultés de l'action publique locale. La seule tentative d'unification à cette époque est à l'initiative de Jules Chevalier, maire 
d'Aulnoy, sous forme d'un district qui aurait pour objectif de mettre en place une agence d'urbanisme. Se heurtant à des oppositions politiques, le projet est rapidement abandonné.

Parallèlement, à la fin des années 1970, se crée un Syndicat Intercommunal à Vocation Unique (SIVU) transports. Rappelons tout d'abord la particularité de cet arrondissement sur le plan de la mobilité. Historiquement, la configuration urbaine caractéristique des cités minières faisait que la main d'œuvre locale se déplaçait peu. Dans les années 1970-1980, le système de transports, fortement structuré par le fonctionnement du système productif local, continue d'être développé en fonction des besoins des entreprises. Ensuite, avec les premiers effets de la crise économique et l'explosion du chômage, les préoccupations du Valenciennois, sont pour l'essentiel liées au développement économique et à l'emploi. Ainsi, le contexte démographique, urbain et social demeure encore aujourd'hui peu favorable à l'émergence d'enjeux forts en matière de développement des transports collectifs.

Jusqu'au milieu des années 1970, l'exploitation du réseau dans le Valenciennois était assurée par la société privée des Chemins de Fer Économiques du Nord. Or, à cette époque, les difficultés financières croissantes l'obligent à abandonner cette exploitation. Comme dans la plupart des autres villes françaises, les dégradations des finances sont liées à la mise en place du cercle vicieux de la diminution des recettes qui entraîne une diminution des investissements, un vieillissement du matériel et une baisse de fréquentation.

En 1974, suite à l'extension de la taxe de Versement Transport aux agglomérations de province de plus de 100000 habitants, le sénateur-maire de Valenciennes, Pierre Carous, prend l'initiative de réunir plusieurs maires de l'arrondissement pour constituer un syndicat intercommunal des transports. Jules Chevalier, maire d'Aulnoy-lez-Valenciennes, est informé de la préparation de la loi sur le Versement Transport ${ }^{8}$. Comme de nombreuses agglomérations de province ${ }^{9}$, le rôle du Versement-transport dans la dynamique intercommunale du Valenciennois est capital. La création d'un organisme intercommunal est obligatoire pour atteindre les 100.000 habitants et percevoir le versement transport, sachant que la commune de Valenciennes ne compte « que » 42000 habitants en 1975.

Le Syndicat Intercommunal des Transports Urbains de Valenciennes (SITURV) est alors créé en 1976, sur un périmètre de 28 communes autour des villes les plus importantes de l'arrondissement. Pierre Carous ayant refusé de présider le SITURV, soulignant la faible implication de la ville centre sur les questions de transports collectifs, Georges Bustin, maire du Vieux Condé, devient le président de l'autorité organisatrice, largement assisté par le premier vice-président, Jules Chevalier.

Très vite, Jules Chevalier s'intéresse avec François Troget $^{10}$, à la création d'une société d'économie mixte qui serait chargée de l'exploitation du réseau de transports urbains. La SEMURVAL ${ }^{11}$ est alors créée en 1979. Elle est présidée par le Maire d'Aulnoy, Jules Chevalier. L'implication directe de ce dernier dans la présidence des deux structures marque alors le début d'une période caractérisée par une symbiose totale entre l'autorité organisatrice

\footnotetext{
${ }^{8}$ Entretien avec Jules Chevalier, maire d'Aulnoy.

${ }^{9}$ Entre 1975 et 1982, 44 Autorités organisatrices vont voter la mise en place du Versement-Transport, soit en créant un organisme intercommunale, soit en s'associant à d'autres communes pour atteindre le seuil de perception de la taxe fixée à 100000 habitants. Voir : Menerault, 1993.

${ }^{10}$ François Troget est ingénieur d'arrondissement de la DDE ; il a assisté le président du Sénat (M.Poher) lors de la préparation de la Loi sur le Versement Transport.

${ }^{11}$ Société d'Économie Mixte des Transports Urbains de la Région de Valenciennes.
} 
(le SITURV) et l'exploitant (la SEMURVAL). De la fin des années 1970 aux années 1990, le système organisationnel de la politique des transports publics dans le Valenciennois est particulièrement stable.

Cette phase de construction intercommunale est marquée par le cloisonnement de la compétence transport lié notamment à l'affectation d'une ressource de financement propre. Parallèlement à l'essor d'une coopération sectorisée dans le domaine des transports publics urbains, que l'on peut rapprocher d'une «intercommunalité de tuyau » ${ }^{12}$ (Dall'Aglio, Petitet, 2000), les réformes territoriales marquent un coup d'arrêt. Jusqu'à 1992 avec la loi ATR, peu de nouveaux textes tentent de renforcer la coopération entre communes ${ }^{13}$.

\section{Figure 2 : L'extension progressive du Périmètre des Transports Urbains dans le Valenciennois entre 1976 et 2004}

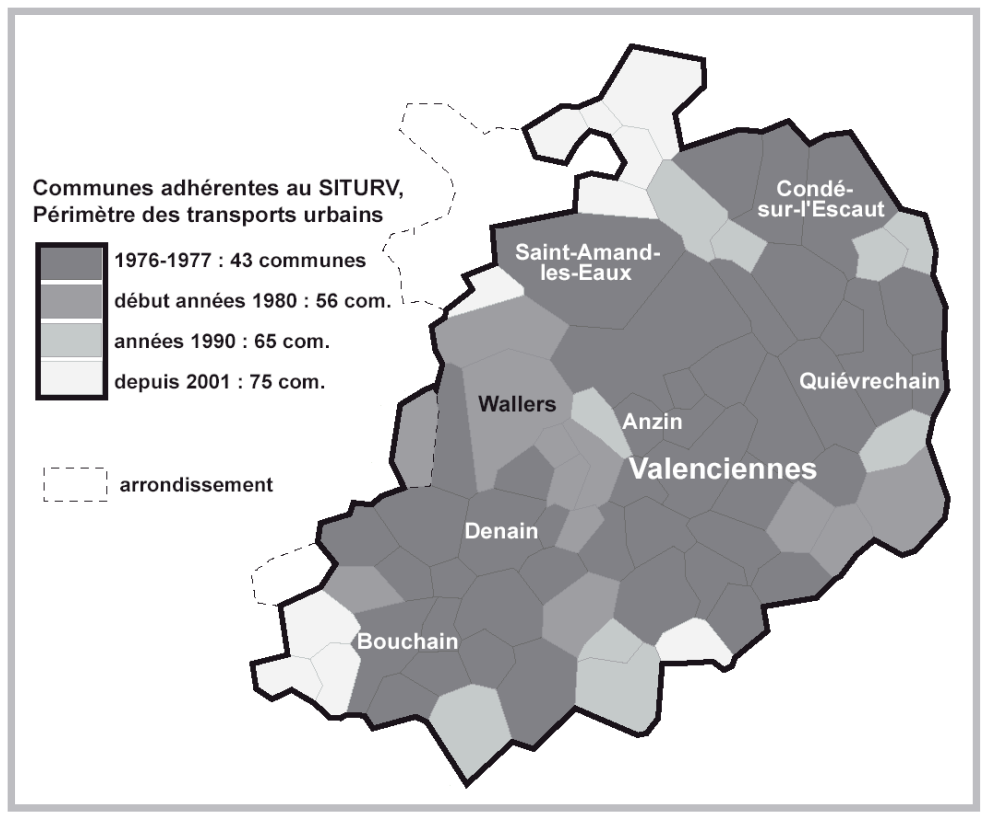

L'assise territoriale de l'intercommunalité en matière de transport urbain dans le Valenciennois s'est accrue progressivement depuis sa création par l'adhésion de communes supplémentaires. A l'origine composé de 28 communes, le périmètre des transports urbains (PTU) s'est rapidement étendu: en 1979, année de la création de la SEMURVAL, il regroupait 51 communes, soit 315000 habitants. Contrairement à de nombreux PTU qui sont restés stables, celui de Valenciennes s'entend encore par la suite, jusqu'à atteindre 65 communes en 1989. L'espace de gestion des transports urbains semble tendre vers les limites de l'arrondissement (figure 2), qui apparaît alors comme le référentiel fonctionnel dans le Valenciennois, en regard des documents de planification élaborée à cette échelle.

Pourtant, suite à l'adoption de la Loi ATR en 1992, les transformations institutionnelles conduisent à un important morcellement intercommunal.

\footnotetext{
${ }^{12}$ D'après les auteurs, «l'intercommunalité de tuyau » désigne une coopération « fonctionnelle », « de gestion » qui concerne les réseaux et équipements collectifs et qui « contribue à construire une solidarité physique ».

${ }^{13} \mathrm{La}$ 《parenthèse intercommunale» fait suite à une phase autoritaire (Loi Marcellin sur les fusions de communes en 1971) qui a alimenté une crainte de l'intégration intercommunale. Voir Menerault, 2005.
} 


\section{De la loi ATR (1992) à la loi Chevènement (1999) : Un projet de transports publics à l'échelle de l'agglomération ... confronté à la « balkanisation » de l'arrondissement}

Cette deuxième période, marquée par une transformation de la dynamique intercommunale, intervient dans les années 1990, suite au vote de la loi relative à l'Administration Territoriale de la République de 1992. La création des communautés de communes provoque un morcellement très important de l'arrondissement alors que, parallèlement, un projet de développement du réseau concernant l'ensemble de l'agglomération et s'appuyant sur un Transport Collectif en Site Propre (TCSP) voit le jour. La question se pose quant à la manière dont ce projet peut s'intégrer dans ce paysage intercommunal très fragmenté et composer avec une scène d'acteurs dans laquelle les affinités partisanes pèsent encore fortement.

\subsection{La «balkanisation» de l'arrondissement (1992-1993) comme révélateur des tensions locales.}

A la suite du vote de la loi ATR, une fièvre intercommunale s'empare de l'arrondissement et concoure à son morcellement exceptionnel. Un an après l'adoption de la loi, le Valenciennois est structuré autour de 9 établissements intercommunaux à fiscalité propre (puis 8 avec l'intégration du SIVOM de Quiévrechain dans la CCVE, figure 3). Les multiples créations de communautés de communes s'effectuent largement en fonction des affinités partisanes avec « des limites qui défient toutes logiques urbaines » (Subra, 1996).

Figure 3 : La création des communautés de communes à la suite de la loi ATR (1992)

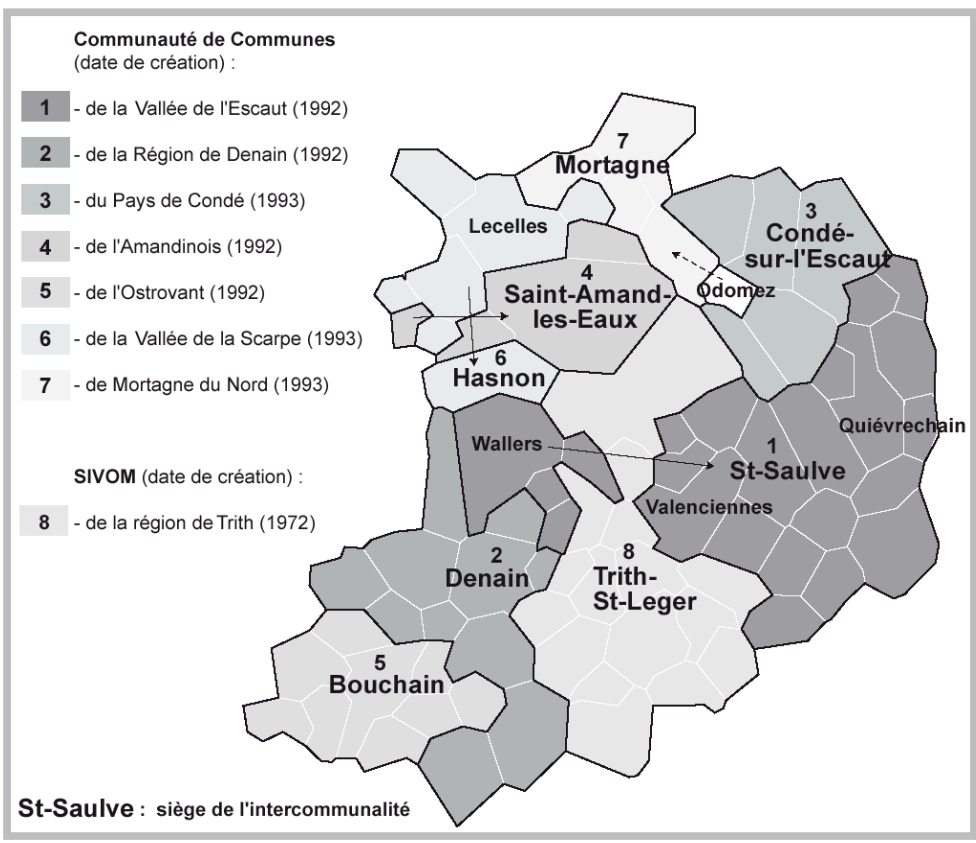

Ainsi en décembre 1992, la communauté de communes de la Vallée de l'Escaut est créée, regroupant, autour de Valenciennes, 19 communes (soit 128000 habitants). Elle est présidée par Cécile Gallez, maire de St-Saulve. Parallèlement, d'autres structures intercommunales se créent (les communautés de communes de l'Ostrevant, de l'Amandinois, de la vallée de la Scarpe, du Pays de Condé et de Mortagne du Nord) ou se transforment (le SIVOM de Denain devient une communauté de communes et le SIVOM de Quiévrechain rejoint la communauté de communes de la Vallée de l'Escaut en 2000). 
Le schéma intercommunal de l'arrondissement apparaît alors "particulièrement confus » (Subra, 1996). Cette balkanisation du Valenciennois, qui perdure jusqu'à la fin de l'année 2000, résulte de la reprise en main du développement territorial par les acteurs politiques locaux. Dans ce contexte, le rôle de l'État demeure important, "notamment sur les registres de l'impulsion, sur celui de la mise en forme et sur celui de l'encadrement du dispositif » (Menerault, 2005). Néanmoins, l'initiative est laissée à l'élu local qui détient la possibilité de former des groupements discontinus ${ }^{14}$. Leurs prérogatives se trouvent ainsi renforcées, alors que, pendant longtemps, dans le Valenciennois, on a observé une vacance du pouvoir politique au profit des houillères ou des grandes firmes sidérurgiques.

Avec la loi Joxe débute une nouvelle phase de réformes territoriales fondée sur une intercommunalité de projet. A partir de 1992, les dispositifs législatifs qui vont se succéder, visent à la fois à renforcer la cohérence spatiale et la cohérence intersectorielle, en s'appuyant sur la notion de projet. Cette forme d'intercommunalité laisse l'initiative au local et sert paradoxalement à pérenniser l'architecture communale (Vanier, 2004). La participation active de l'élu local à l'élaboration des découpages intercommunaux implique l'expression de clivages partisans qui débouche souvent sur la constitution de véritables «fiefs politiques ». L'expérience intercommunale du Valenciennois en fournit une illustration saisissante (Cf figure 3).

\subsection{Un réseau de transport public atone ... mais un projet tramway ambitieux}

La question est alors de savoir comment le fonctionnement du réseau de transport et la structuration de l'autorité organisatrice composent avec ce paysage intercommunal ? Quelle influence exerce cette configuration éclatée sur le traitement des enjeux de transport public orienté vers un projet de tramway dans l'arrondissement valenciennois?

La spécificité du PTU valenciennois est d'englober autant de communes rurales que de communes urbaines ${ }^{15}$. En outre, comme nous l'avons mentionné précédemment, plusieurs éléments semblent assez peu favorables à l'émergence d'enjeux forts de transports publics dans cette agglomération. La dispersion de la population sur l'arrondissement ne favorise pas le développement de nouvelles lignes de Transport Collectif (TC). Lorsqu'on compare le réseau Valenciennois aux autres agglomérations dont la population du PTU est à peu près équivalente, il apparaît que l'offre et la fréquentation du réseau sont relativement faibles. Le comparatif de la fréquentation entre les agglomérations qui comptent entre 200000 et 500000 habitants (figure 4) montre clairement la faiblesse des réseaux de transport public de l'ancien bassin minier du Nord-Pas-de-Calais. Par ailleurs, les résultats médiocres du réseau Valenciennois sont à mettre en relation avec l'omniprésence de l'automobile qui profite de bonnes conditions de circulation avec un réseau d'infrastructures routières et autoroutières bien développé. Enfin, la morphologie urbaine particulière, héritée de l'ère industrielle, avec un bâti linéaire peu densifié, est peu aisée à desservir en transport collectif et génère des coûts d'exploitation supplémentaires.

\footnotetext{
${ }^{14}$ Une liberté qui sera réduite par la loi Chevènement. Le législateur prend acte des effets de la loi Joxe et incite alors à créer des structures intercommunales « d'un seul tenant et sans enclave ».

${ }^{15}$ Cette caractéristique résulte du choix de l'autorité organisatrice de laisser « ouvert » l'adhésion au syndicat des transport pour toutes les communes de l'arrondissement et pas seulement les plus urbaines.
} 


\section{Figure 4 : Comparatif de la fréquentation entre les réseaux français compris entre 200000 et 500000 habitants (en nombre de voyages par habitants, 2002). Source : annuaire statistique GART, CERTU, DTT, UTP.}

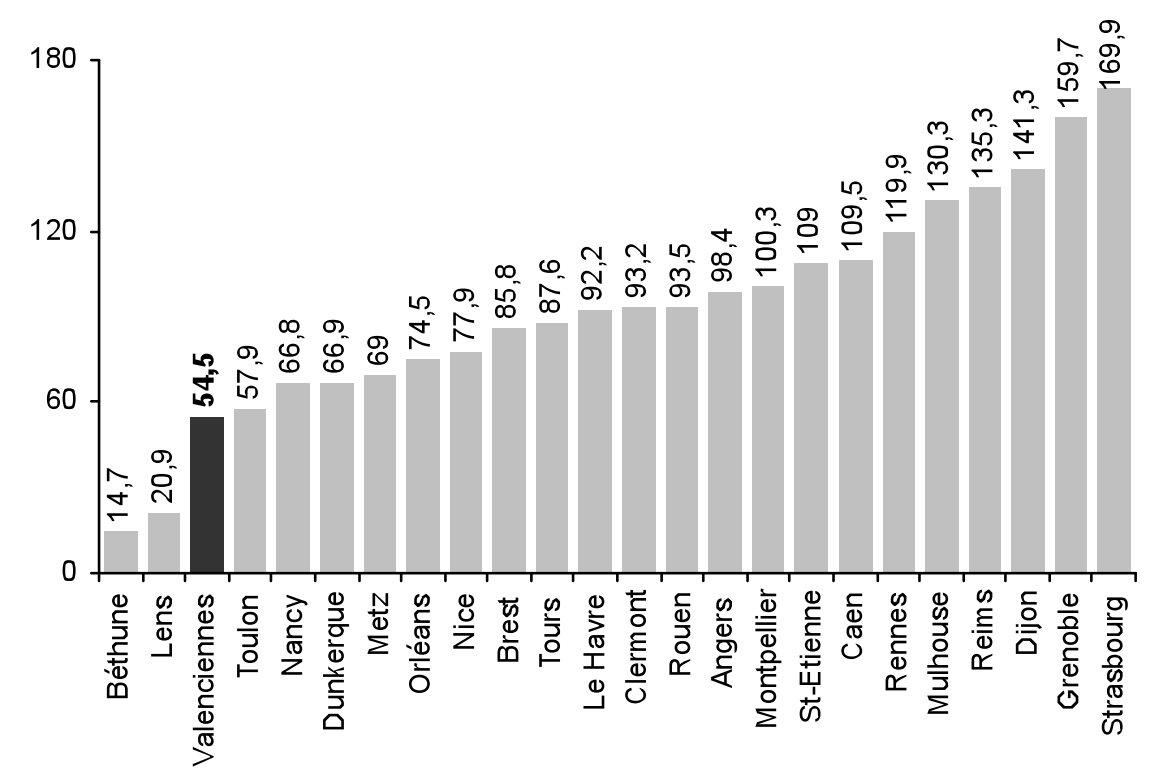

Jusqu'aux années 1960, les transports publics valenciennois disposaient encore d'un important réseau de tramway qui a progressivement laissé sa place à l'autobus à l'instar de nombreuses agglomérations françaises. Les évolutions à la baisse de la clientèle des transports collectifs, les problèmes des temps de transport et les limites de capacité (versement transport limité) ont encouragé la recherche de solutions. Ainsi, c'est dans le contexte d'un réseau de transports collectifs qui s'essouffle et à travers la volonté de le redynamiser que l'idée de construire un nouveau tramway va se forger.

Au congrès de Brest (concours du tramway français moderne en 1977), le président du SITURV est convaincu de l'utilité d'un tramway pour Valenciennes. En 1991, l'INRETS, par l'intermédiaire de l'étude Vulcain ${ }^{16}$, démontre que Valenciennes a le niveau et le besoin d'un Transport Collectif en Site Propre (TCSP). Plusieurs matériels sont envisagés mais le métro léger de type Val n'est pas concevable sur le plan financier. La solution du tramway semble alors mieux adaptée : "l'examen technologique, face aux contraintes et aux possibilités financières, fait ressortir le choix du tramway comme le mode répondant le mieux à toutes les questions ${ }^{17}$, précise le dossier de prise en considération ${ }^{18}$ déposé au Ministère des Transports. Le projet, baptisé «Transvilles », prévoie à l'échelle de l'agglomération une réorganisation des transports publics s'appuyant à la fois sur la réalisation d'un tramway moderne et un redéploiement des lignes de bus.

L'évolution chaotique du dossier illustre le cloisonnement des institutions et les positions divergentes entre l'exploitant, à l'origine du projet, et les élus de la ville-centre, qui y sont assez réticents. Du côté de la SEMURVAL, en étroite collaboration avec l'autorité

\footnotetext{
${ }^{16}$ Étude sur un métro de type Val dans une agglomération moyenne. Elle a été réalisée à la demande du SERT par l'INRETS-CRESTA, METRAM et le CETUR.

${ }^{17}$ La solution tramway est envisagée pour les «barreaux 1 à 4 » qui forment un H. Il s'agit des liaisons entre Anzin et Musée (barreau 1), Musée et Université (barreau 2), Musée et St-Saulve (barreau 3), Anzin et Dutemple (barreau 4).

${ }^{18}$ SITURV, 1993.2001, le réseau Bus-Tram dans le Valenciennois. Dossier de prise en considération, 84 p.
} 
organisatrice, le projet est déjà engagé techniquement d'autant plus que l'exploitant bénéficie de l'expertise du groupe TRANSDEV en matière de TCSP. En 1994, le comité syndical du SITURV choisit le matériel puis l'avant-projet est accepté. Un an plus tard, la concertation publique démarre. Le ministère de l'Équipement annonce, en 1997, la prise en considération par l'Etat de la première phase du projet Transvilles. Le comité syndical valide ensuite la réalisation de la première phase du programme.

Mais parallèlement, le dossier est vivement contesté. En 1997, l'association Vigitramway est créée dans l'Amandinois pour s'opposer au projet. Celle-ci considère que Transvilles est trop coûteux, et qu'il pénalise les entreprises ${ }^{19}$. Au sein de l'association, les commerçants et riverains s'opposent également au projet, ce qui explique les hésitations des élus valenciennois pour valider un tracé sur leur commune. En outre, au sein même du comité syndical du SITURV, la commune de Marly est opposée et St Amand est réservée. D'autre part, le tramway est perçu par la ville-centre comme un projet du SITURV et de la SEMURVAL, donc imposé par des élus politiquement opposés à Valenciennes.

Transvilles fait l'objet d'âpres négociations, bien illustrées par les multiples évolutions du tracé du tramway (Figure 5). Celui-ci résulte d'un savant compromis entre les différents acteurs. D'autre part, en dépit du nom du projet «trans-villes », qui suggère que le tramway est un vecteur de solidarités trans-communales renforcées, c'est plutôt l'autonomie communale qui semble préservée, puisque les communes ont choisi individuellement leur tracé. En gérant les réseaux dont les communes ont choisi le tracé (exemple du tramway de Valenciennes), la coopération intercommunale apparaît davantage comme un relais du pouvoir municipal (Brechon-Moulenes, 1988), que comme un véritable échelon supracommunal.

\footnotetext{
${ }^{19}$ Les milieux économiques (chefs d'entreprises, PME, groupement patronal, artisans) réagissent mal au passage du taux de versement transport de 1 à $1,75 \%$, un recours est déposé devant les tribunaux administratifs.
} 
Figure 5 : Les vicissitudes du tracé du Tramway de Valenciennes (1991-2006)

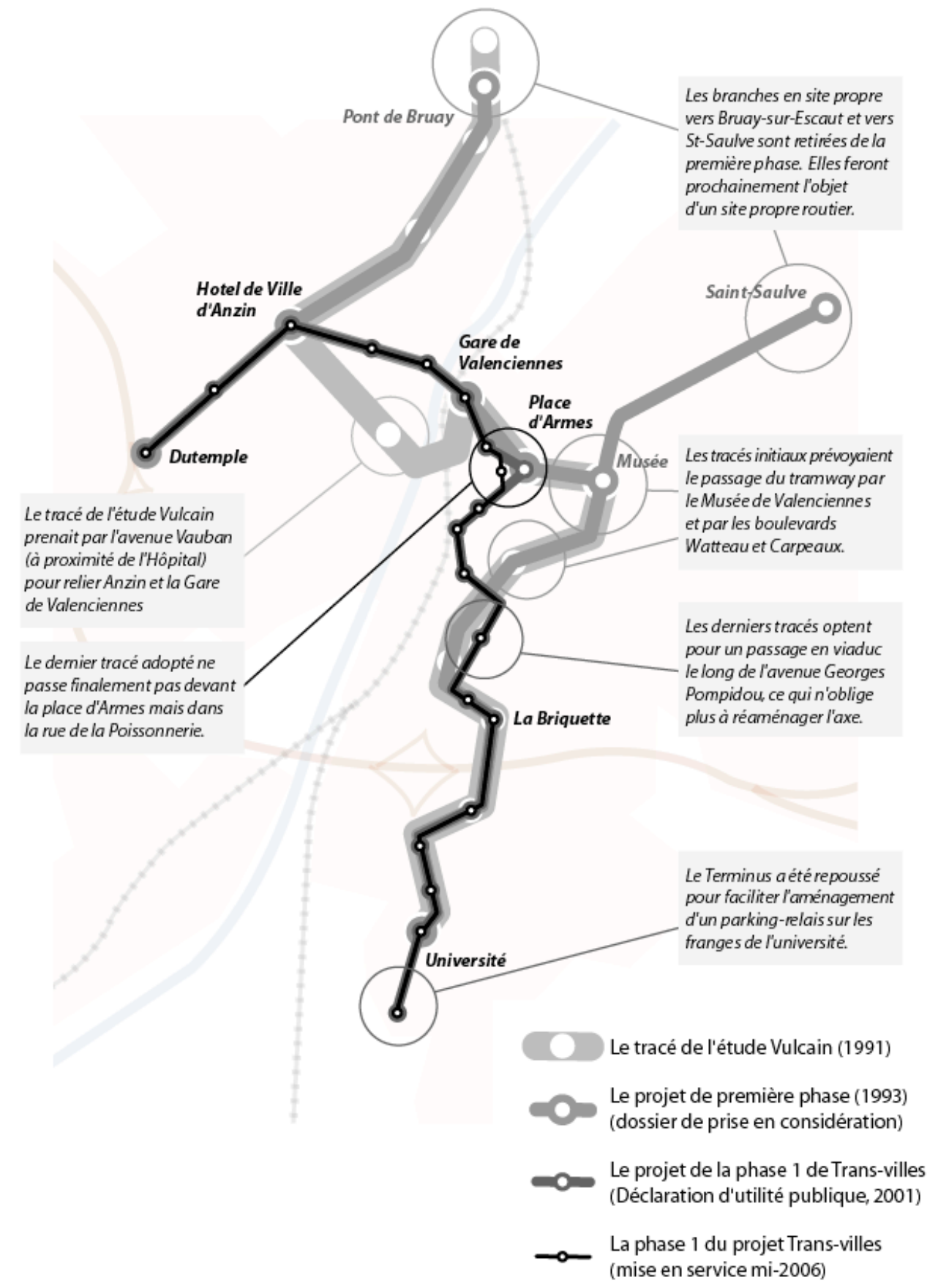

Pour être entériné, le dossier tramway a eu besoin d'une certaine maturation sur le plan politique et surtout de l'adhésion de la ville centre. A l'origine considérée comme un dispositif global de réorganisation des transports urbains à l'échelle de l'agglomération ${ }^{20}$, les élus valenciennois ont progressivement perçu l'opportunité que constituait l'arrivée d'un tramway en tant qu'outil d'urbanisme et de modernité dans une stratégie de redynamisation de la ville. Ce n'est que récemment que les élus valenciennois ont mobilisé le tramway comme un promoteur des politiques de rénovation urbaine.

\footnotetext{
${ }^{20}$ Pour l'exploitant, le projet conserve la dimension de projet de transport à l'échelle de l'agglomération puisque, depuis 2006, le réseau de transport urbain de la SEMURVAL a adopté le nom de Transvilles.
} 
Malgré le changement d'image de la ville souhaité par Jean-Louis Borloo depuis son premier mandat, les réflexions accompagnant le projet de tramway ont été, pendant longtemps, scindées de la politique urbaine développée à Valenciennes autour d'une chaîne de réalisations importantes ${ }^{21}$.

Les négociations intervenues en parallèle de l'élaboration du Plan de Déplacements Urbains (PDU) traduisent bien le résultat d'un compromis et d'un arbitrage entre les acteurs valenciennois et l'autorité organisatrice. En effet, les élus de la ville centre ont considéré pendant longtemps le TCSP comme un mode de transport en contradiction avec une politique d'attractivité commerciale. Un extrait du registre des délibérations de la ville de Valenciennes de mai 1999 illustre particulièrement bien la politique de compromis pratiquée entre les deux parties : les élus valenciennois valident le projet de tramway dans le cadre de l'élaboration du PDU, à condition que ce dernier impulse en compensation le projet valenciennois de rocade de contournement sud de la ville, ainsi que l'implantation de parcs de stationnement. Ainsi, à l'issue de nombreuses discussions, le vote définitif du tracé du tramway sur la commune de Valenciennes intervient au printemps 1999.

Depuis 2002, le ministère de l'Équipement a donné un avis favorable à la demande de subvention, qui a permis au SITURV de boucler son plan de financement et de démarrer les travaux (figure 6). L'inauguration de la première phase du tramway a eu lieu le 16 juin 2006.

\section{$\underline{\text { Figure } 6}$ : Le chantier du tramway de Valenciennes (station Clemenceau, janvier 2006)}

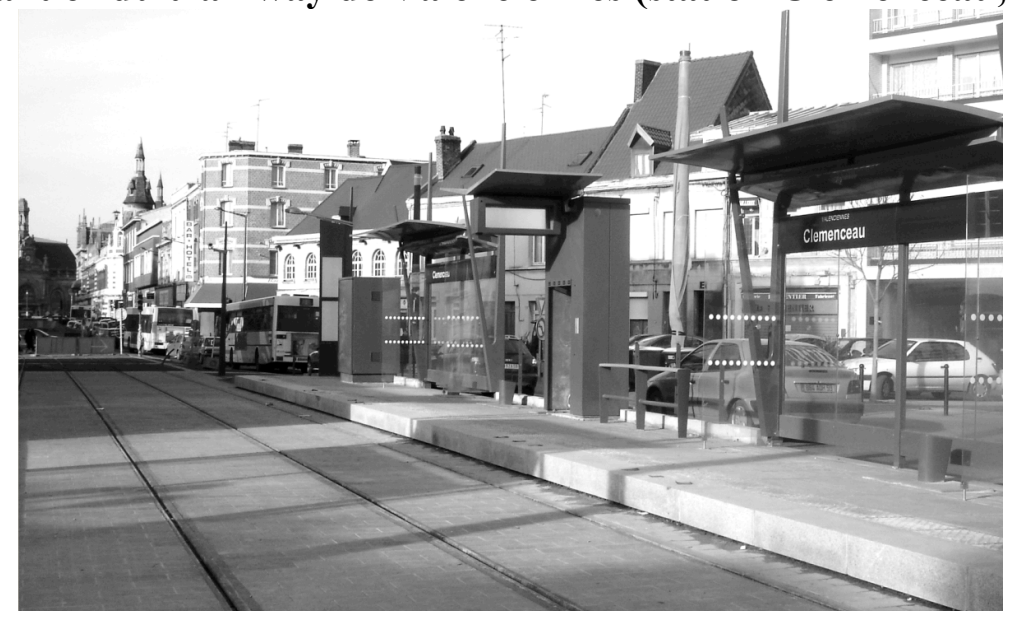

\subsection{Une absence de transversalité chronique couplée à l'impossible affirmation d'une structure d'arrondissement}

Alors que le projet a été entériné et validé politiquement, celui-ci apparaît comme un projet technique complètement scindé d'une réflexion stratégique sur les déplacements de l'agglomération valenciennoise. En outre, ce projet a vu le jour et a évolué dans un contexte où le schéma intercommunal très éclaté ne favorisait ni les solidarités internes ni les réflexions stratégiques à l'échelle de l'agglomération.

Ainsi, parallèlement à l'organisation institutionnelle morcelée, se met en place une autre forme de partenariat à l'échelle de l'arrondissement qui rassemble l'ensemble des communes de l'arrondissement : l'Association pour le Développement du Valenciennois (ADV), créée en

\footnotetext{
${ }^{21}$ Par exemple : le projet « Coeur de Ville », le théâtre du « Phénix », le musée du Hainaut...
} 
1991. Son rôle consiste alors à assurer la représentation économique de l'arrondissement. C'est un article dans le contrat de plan de l'époque qui incitait les territoires à se regrouper pour élaborer des projets d'agglomération puis des contrats d'agglomération qui a motivé la création de cette association. Il y avait donc une incitation financière. Ainsi l'association a porté le projet d'agglomération de Valenciennes et s'est engagée dans les procédures de contractualisation avec l'Etat et la région. Elle a été la seule en France à avoir signé un contrat de plan.

Au début des années 1990, les tentatives de transformation de l'association en SIVOM à la carte ou en syndicat mixte, portées par les maires d'Anzin et d'Aulnoy, échouent devant la crainte des élus des communes rurales d'un renforcement du degré d'intégration de la coopération intercommunale. De leur côté, Jean-Louis Borloo (divers Droite) et Alain Bocquet (PC), nouvellement élus maires respectivement de Valenciennes et de Saint-Amand, craignent de voir le rôle d'un élu socialiste ainsi renforcé. La souplesse du statut associatif et le principe d'une "présidence tournante » pour l'ADV rassurent en revanche l'ensemble des élus. Le fonctionnement découpé en quatre zones de cette association traduit également les clivages. Le projet d'agglomération de 1994/1998 prévoyait que l'association laisse ensuite la place à une structure plus forte, aux compétences élargies.

Présentée comme le seul élément d'unité dans les années 1990, l'association n'est en fait qu'un édifice fragile, marqué par les clivages politiques. Le manque d'ambition intercommunal et les clivages partisans empêchent l'ADV de déboucher sur une structure intercommunale unique. La coopération à l'échelle de l'agglomération continue à effrayer et conduit la majorité des élus à défendre les intérêts communaux qu'ils représentent (Gaxie, 1997) au lieu d'être les animateurs d'une institution distincte.

Mais là encore les clivages ne sont pas dépassés. Ces diverses oppositions de principe ou sur le choix de la forme et des compétences d'une intercommunalité à l'échelle de l'arrondissement préfigurent les difficultés qui surgissent un peu plus tard, après le vote de la loi Chevènement. 


\section{Depuis la loi Chevènement (1999) : Du «mirage intersectoriel » à la préservation du PTU : les effets controversés de l'exacerbation des leaderships politiques}

La troisième période débute après l'adoption de la loi Chevènement relative au renforcement et la simplification de la coopération intercommunale adoptée en 1999. La réforme pose à nouveau la question de la structuration intercommunale du Valenciennois. Comment l'ambition législative réaffirmée de cohérence spatiale et intersectorielle peut-elle trouver une traduction dans le Valenciennois ? Dans cette refonte institutionnelle, les politiques de transport urbain entraînées par l'aboutissement du projet tramway peuvent-elles devenir le substrat territorial de solidarités intercommunales renouvelées? Une fois encore, la question $\mathrm{du}$ leadership politique et les clivages partisans dans cet arrondissement ne sont-ils pas susceptibles de freiner ces ambitions?

\subsection{La création des deux communautés d'agglomération : une « coupure » durable de l'arrondissement ?}

Alors que différents partis politiques et conseils municipaux se prononçaient en faveur de la constitution d'une communauté d'agglomération à l'échelle de l'arrondissement et que les représentants de l'Etat tenaient le même discours, deux communautés d'agglomération distinctes se sont mises en place : «Valenciennes Métropole» regroupant 36 communes et présidée par Jean-Louis Borloo ${ }^{22}$ (environ 200000 habitants) et «La Porte du Hainaut» présidée par Alain Bocquet ${ }^{23}$ (150 000 habitants) et composée de 38 communes (figures 7 et 8). Elles résultent de longues négociations qui ont d'abord opposée ${ }^{24}$ puis associé les deux leaders politiques contre la volonté du préfet. Malgré le caractère concurrentiel de leur entreprise politique, Alain Bocquet et Jean-Louis Borloo sauront s'unir pour éviter la mobilisation des autres élus de l'arrondissement autour d'un projet non conforme à leur aspiration, pour négocier avec le Préfet la création au sein de l'arrondissement de deux structures, pour déposer et défendre à l'assemblée nationale quatre amendements à la loi Chevènement, afin de l'adapter à la spécificité de l'arrondissement.

\footnotetext{
${ }^{22}$ Jean-Louis Borloo est maire de Valenciennes depuis 1999, député UDF puis UMP depuis 1993 et ministre sous le gouvernement de Jean-Pierre Raffarin et de Dominique de Villepin.

${ }^{23}$ Alain Bocquet est maire de Saint-Amand-les-Eaux depuis 1992, député depuis 1978 et président du groupe communiste à l'Assemblée Nationale.

${ }^{24}$ La réunion d'Hasnon (juin 2000) à l'initiative de Saint-Amand concoure à isoler Valenciennes en créant une communauté d'agglomération de 45 communes. Le conseil municipal de Valenciennes répond en présentant le "périmètre évident» pour le projet de création d'une communauté d'agglomération. Les périmètres convoités par les leaders politiques se chevauchent.
} 
Figures 7 et 8 : La structuration intercommunale du Valenciennois suite à la création des deux communautés d'agglomération (2001) et l'organisation politique de l'arrondissement suite aux élections municipales de 2001.

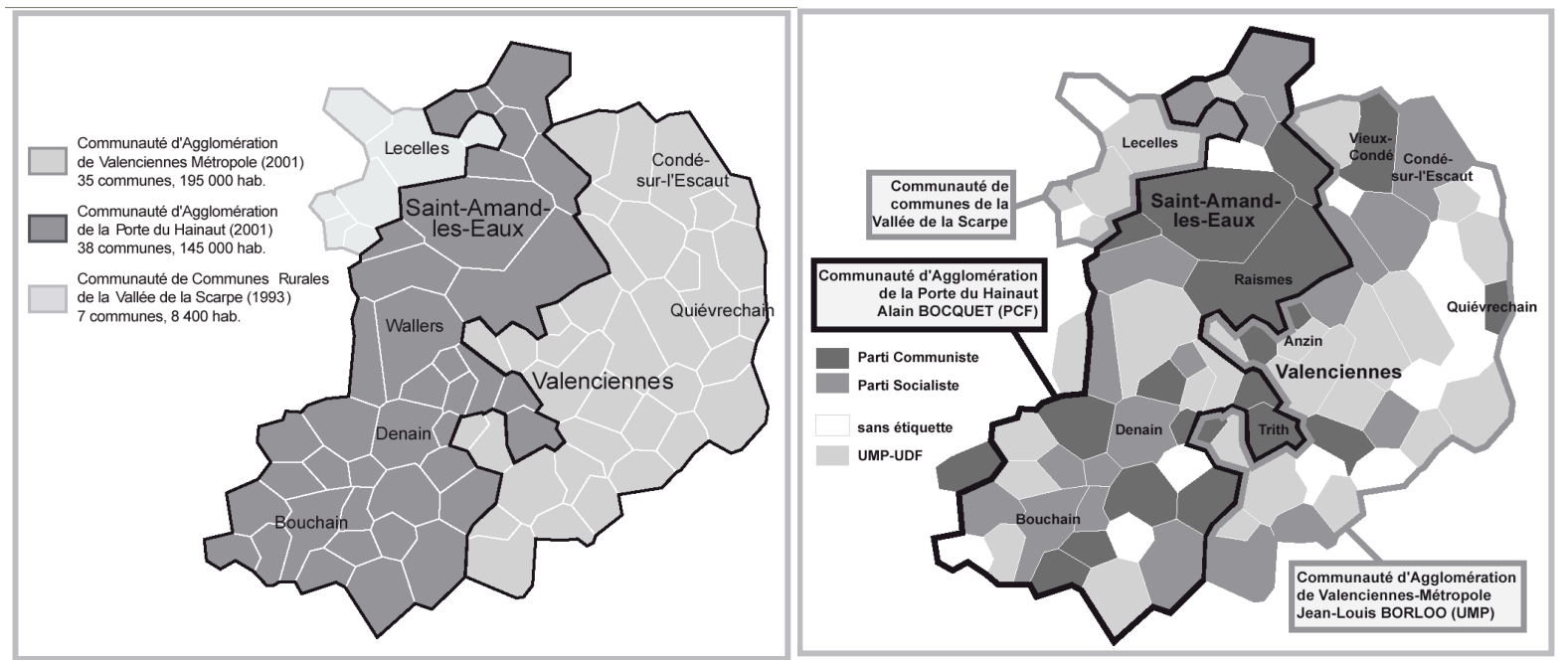

L'activité politique d'Alain Bocquet et de Jean-Louis Borloo se combine entre le local et le national. Ce «double horizon des pratiques ${ }^{25}$ conduit ces élus à appréhender de façon particulière les enjeux de l'intercommunalité. La présidence d'une intercommunalité peut se concevoir comme un outil de consolidation du leadership local et comme une ressource supplémentaire pour peser sur la scène politique nationale.

De nombreux acteurs locaux (en particulier les techniciens) ainsi que la presse présentent cette situation comme le produit d'un découpage politique du Valenciennois, c'est-à-dire comme le résultat du clivage partisan entre gauche et droite d'une part, et de «la constitution des fiefs de deux personnalités politiques d'envergure nationale » ${ }^{26}$, d'autre part. Après discussion et vote des différents élus, les périmètres des deux structures se dessinent finalement en fonction de la répartition égale des ressources fiscales (taxe professionnelle) de part et d'autre.

Alors que la séparation de l'arrondissement en deux communautés d'agglomération semble " contre nature » à certains élus et aux représentants de l'Etat, elle apparaît cohérente à d'autres élus et techniciens dans le fonctionnement. En effet, les différentes politiques contractuelles révèlent un fonctionnement en deux entités autonomes qui font bloc autour de leur projet de territoire, en considération des enjeux financiers liés aux contrats de plan avec la région. La coopération entre les structures opère au cas par cas, sur des procédures dont les enjeux sont faibles ou lorsque le cadre étatique l'impose. L'organisation actuelle semble représenter un compromis entre un "espace de pouvoir » (échelle d'intervention politique) et un «territoire de projet» (échelle de planification et d'aménagement). Cette séparation de l'arrondissement en deux entités distinctes est liée à la manière dont chacun des deux leaders entend élaborer son action publique locale. Les deux communautés d'agglomération apparaissent comme de nouveaux lieux d'appropriation politique des enjeux par les acteurs

\footnotetext{
${ }^{25}$ Cité par Dechy (2001), d'après une expression de Lagroye J., 1993. De l'objet local à l'horizon local des pratiques. in A. Mabileau (dir.), A la recherche du local, Paris, L'Harmattan, pp. 166-182.

${ }^{26}$ D'après Dechy, 2001.
} 
locaux. Cette organisation contraste avec la vision unifiée de l'arrondissement, qui était véhiculée par les représentants de l'État, à une période où les élus locaux étaient en retrait.

\subsection{La transformation du SITURV, dans une logique de «préservation »}

En 2001, le SITURV se transforme en syndicat mixte. La compétence «organisation des transports publics », obligatoire pour les deux communautés d'agglomération, a remis en cause l'existence de l'autorité organisatrice sous sa forme de SIVU. Pour permettre la continuité du service public de transport, et en arguant de la nécessité d'assurer une gestion cohérente du réseau, les deux communautés d'agglomération vont déléguer leur compétence en matière «d'organisation des transports urbains » au SITURV. En février 2001, l'autorité organisatrice modifie ses statuts et se transforme en syndicat mixte ${ }^{27}$ par arrêté préfectoral du 5 juin 2001.

A l'image de l'évolution juridique du SITURV dans le Valenciennois, une quinzaine d'autorités organisatrices françaises ont consenti le même mouvement depuis 1999 (Richer, 2005). La création de ces syndicats mixtes résulte d'une même logique de préservation: elle consiste à maintenir l'unité du réseau de transport public à la suite de la création d'une ou de plusieurs communautés d'agglomération, dont l'assise territoriale n'englobe pas la totalité du périmètre de transport urbain antérieur. Devant la fragmentation du territoire des transports générée, les nouvelles communautés d'agglomération ont délégué leur compétence transport à un syndicat mixte. Paradoxalement, les trois quarts des nouvelles créations de syndicats mixtes depuis 1999 sont liées à la loi Chevènement et non à la loi SRU qui pourtant tente de relancer ce type de formule, avec l'ambition de coordonner différents échelons d'autorités organisatrices urbaines et interurbaines.

Bien que les périmètres de transport soient maintenus, au minimum dans leur composition antérieure, ce processus original et inattendu ne favorise pas l'intégration de la compétence transport dans une configuration institutionnelle plus intégrée et plus transversale. Ces nouveaux syndicats demeurent monovalents et n'associent pas d'autres niveaux d'autorités organisatrices de transports. Cette dynamique marque également l'absence de réflexions préalables à la création des communautés sur la question des transports. L'exemple du Valenciennois montre que les transports publics, jadis promoteurs de solidarités intercommunales, ne sont aujourd'hui plus porteurs de l'évolution des structures de coopération entre communes.

D’une manière générale, la compétence relative à « l'organisation des transports publics » se trouve soit internalisée dans les nouveaux dispositifs législatifs ${ }^{28}$, soit externalisée dans des structures monovalentes de type syndicat mixte ${ }^{29}$. Elle constitue rarement un enjeu fort du développement récent de l'intégration intercommunale. Suite à la loi Joxe (1992), Philippe Menerault constatait : les transports publics, ne sont pas devenus le substrat territorial d'une politique globale de gestion des déplacements dont la cohérence à l'échelle des agglomérations n'a pu s'imposer comme vecteur de solidarités intercommunales renouvelées (Menerault, 1995). Après la loi Chevènement, la même analyse s'impose.

\footnotetext{
${ }^{27}$ Le SITURV, syndicat mixte de transport, se compose désormais de la communauté d'agglomération de Valenciennes Métropole, de la communauté d'agglomération de la Porte du Hainaut et d'une commune, extérieure à l'arrondissement, Hornaing.

${ }^{28}$ La compétence « organisation des transports publics » est intégrée dans le champs de compétence relatif à « l'aménagement de l'espace communautaire » pour les communautés d'agglomération.

${ }^{29}$ Voir sur ce point les logiques adaptatives et intégratives (Gallez, 2005).
} 
Dans le Valenciennois, l'une des premières conséquences de cette configuration institutionnelle est la disparition du «mirage de la globalité» (Offner, 2002). Chacune des deux communautés d'agglomération déploie, selon ses objectifs, son propre arsenal de procédures (contrats d'agglomération et projet de territoire) tandis que le syndicat mixte des transports assure la maîtrise d'œuvre du Plan de Déplacements Urbains. La définition du périmètre du (ou des) futurs SCOT demeure aujourd'hui incertaine. L'élaboration de deux SCOT au niveau des deux communautés d'agglomération se ferait au détriment de la cohésion spatiale du schéma. Néanmoins un SCOT élaboré à l'échelle de l'arrondissement, dans le prolongement des schémas directeurs, n'éliminerait ni le risque d'un effet frontière dû au découpage des structures administratives, ni celui d'une juxtaposition des éléments de diagnostic au détriment d'une cohésion intersectorielle.

\subsection{Une action publique élaborée en « circuit fermé »}

Aujourd'hui, la création des deux communautés d'agglomération oblige le SITURV, outre son changement de statut, à repenser son mode de fonctionnement et son schéma décisionnel pour jouer un rôle de médiateur. C'est dans cette optique que Francis Decourrière ${ }^{30}$ a été élu président du syndicat mixte en juin 2001. Il doit assurer en permanence le consensus entre les deux présidents des communautés d'agglomération notamment sur le dossier transvilles qu'il doit porter politiquement dans sa phase ultime. Ce changement de présidence marque la fin de la symbiose qui caractérisait les relations entre l'autorité organisatrice et l'exploitant. Le SITURV reprend la maîtrise d'ouvrage de Transvilles qui jusque-là avait été portée par la SEMURVAL.

Le fonctionnement du SITURV s'est néanmoins complexifié suite au découpage en deux communautés d'agglomération. Cette configuration ne simplifie pas le processus décisionnel qui est ralenti par une consultation préalable des deux structures. Restant une structure monovalente, le SITURV se trouve en quelque sorte instrumentalisé et doit composer en permanence avec les deux communautés d'agglomération sur les thématiques connexes aux transports publics, aménagement, urbanisme, stationnement... ${ }^{31}$ Les investissements liés au projet «Transvilles» posent la question d'une offre de service équilibrée entre les deux communautés d'agglomération: ces dernières participent de manière équivalente au financement du SITURV tandis que, dans une logique de centralité, les investissements se portent d'abord sur le territoire de Valenciennes Métropole qui accueillera la phase $1 \mathrm{du}$ TCSP sur son territoire. En termes de compensation, la Communauté d'Agglomération de la Porte du Hainaut, devrait alors bénéficier d'une desserte adaptée, du type «transport à la demande $»$.

On assiste à Valenciennes à un cloisonnement encore important entre les structures et leurs acteurs. Le contexte historique et social de cet arrondissement en fait un territoire sur lequel les principaux enjeux pour la qualité de vie reposent sur l'économie et l'emploi. La structuration récente de l'intercommunalité en deux entités distinctes amène à repenser les questions de régulation interne au territoire entre les deux communautés d'agglomération ainsi que celle des enjeux de gouvernement urbain en termes de coordination institutionnelle. Les débats intervenus au lendemain de la Loi Chevènement sur la structuration des deux communautés d'agglomération illustrent les rivalités de pouvoir entre acteurs politiques s'exerçant sur un territoire. Ils montrent également que la décision prise repose finalement sur

\footnotetext{
${ }^{30}$ Francis Decourrière est conseiller municipal à Valenciennes et député européen.

${ }^{31}$ Entretien avec F. Decourrière, Président du SITURV.
} 
le Préfet et deux « grands élus » de l'arrondissement. Pourtant, la discussion sur la nouvelle configuration concernait également les acteurs investis précédemment dans les structures intercommunales, les Chambres consulaires ... Ainsi, loin de repenser les modalités de l'action publique en terme de gouvernance, dans cet arrondissement, l'action publique, élaborée " en circuit fermé », confirme au contraire des modalités classiques de gouvernement reposant entre les mains du préfet et de ses notables, dont les enjeux résident sans doute dans la recherche d'une dynamique et d'une solidarité internes à l'agglomération ${ }^{32}$.

\footnotetext{
${ }^{32}$ Frère, 2005.
} 


\section{Conclusion}

Le paradoxe majeur de l'intercommunalité dans le Valenciennois tient à la difficulté qu'ont les communes à se regrouper en une structure unique d'arrondissement, alors que l'ampleur des problèmes posés par le déclin économique et la reconversion imposerait une solide coopération. Dès les années 1970, les premiers jalons de l'intercommunalité sont posés sous l'impulsion de la DDE, mais le poids des logiques partisanes a toujours fait échouer les différentes tentatives d'unification : la tentative d'unification de l'arrondissement sous forme d'un district ; les propositions de transformations de l'ADV en SIVOM à la carte ou en syndicat mixte au début des années 1990 ; l'absence de création d'une communauté d'agglomération à l'échelle de l'arrondissement en 1999; l'impossible émergence d'une agence d'urbanisme du Valenciennois.

En outre, la vision de l'intercommunalité est restée essentiellement utilitaire, d'autant plus que les syndicats intercommunaux préexistants aux communautés d'agglomération étaient souvent monovalents. Ces structures n'ont donc pas contribué à préparer les acteurs politiques à une forme plus intégrée de l'intercommunalité. Ainsi, l'histoire cumulative ${ }^{33} \mathrm{du}$ Valenciennois, héritée du passé minier et de la crise sidérurgique, semble avoir renforcé les élus dans l'exercice de leur pouvoir et la défense de leurs prérogatives vis a vis de structures intercommunales auxquelles le transfert de compétences est encore perçu comme une dépossession.

Des travaux réalisés antérieurement sur cet arrondissement ${ }^{34}$ ont déjà montré la difficulté à y mettre en place une politique urbaine cohérente, qui serait liée à un manque d'outils d'études communs. Cela renvoie à la question de l'expertise et de l'ingénierie mobilisées sur ce territoire. Dans les années 1970/1980, l'arrondissement a bénéficié de travaux menés dans le cadre du GEP. L'absence d'un relais technique à l'échelle locale expliquerait les difficultés à exploiter l'expertise accumulée pour la mise en œuvre d'une réflexion transversale. La création d'une agence d'urbanisme annoncée depuis une dizaine d'années apparaît encore aujourd'hui comme une tentative avortée bien qu'un directeur ait été recruté. Pourtant, celleci avait été identifiée par Philippe Subra comme un enjeu essentiel pour le renouveau du Valenciennois : «on voit mal comment l'arrondissement pourrait mettre en place, sans outil d'études commun, une politique urbaine cohérente. La création d'une agence de développement économique et d'une agence d'urbanisme est donc indispensable ${ }^{35}$. Cet instrument d'expertise supracommunal suscite la méfiance des élus locaux et interroge les modalités de fonctionnement avec les deux communautés d'agglomération. La question de l'ingénierie et du portage des projets territoriaux dans le Valenciennois semble pourtant particulièrement stratégique pour les années à venir en regard des nouvelles modalités de contractualisation des territoires envisagées par les institutions régionales ${ }^{36}$. Comment le Valenciennois se positionnera-t-il face à ces nouvelles modalités d'action publique?

Une deuxième caractéristique de cet arrondissement tient à la faiblesse de l'enjeu des déplacements. En effet, la dynamique intercommunale dans le valenciennois ne concoure pas à mettre au centre des enjeux les préoccupations liées aux transports. Les transformations de l'intercommunalité, y compris à la suite de la loi Chevènement, n'ont pas donné lieu à l'intégration sectorielle escomptée des transports publics. Ceux-ci conservent une

\footnotetext{
${ }^{33}$ Expression de D. Gaxie, 1997.

${ }^{34}$ Subra, 1996.

${ }^{35}$ Subra, 1996.

${ }^{36} \mathrm{Cf}$ sur ce point, le SRADT de la Région Nord Pas de Calais.
} 
organisation territoriale autonome qui ne garantie pas la transversalité entre les politiques de déplacements et d'urbanisme (Figure 10).

\section{Figure 9 : Schématisation des relations entre l'intercommunalité et la dynamique des transports publics dans le Valenciennois.}

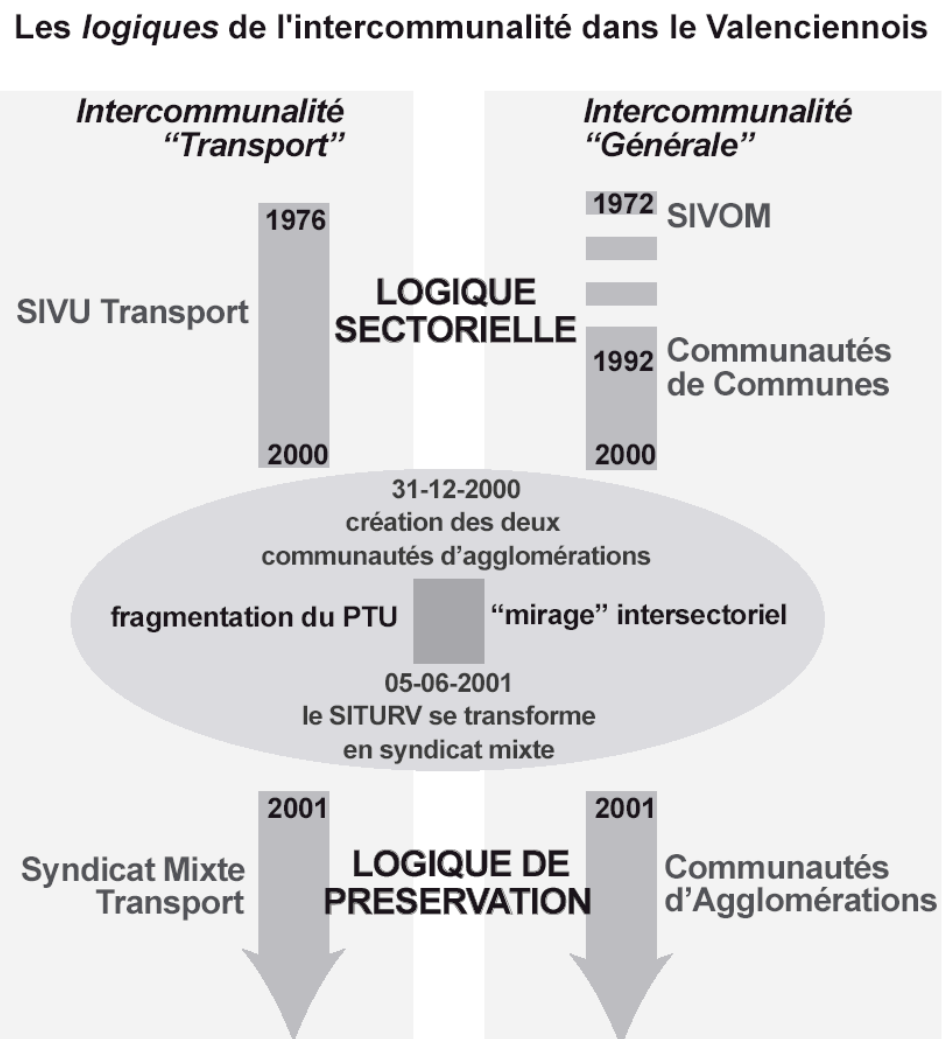

Mais l'enjeu de l'intercommunalité et des politiques de transport dans le Valenciennois, semble se situer davantage au niveau de l'articulation entre les différentes configurations institutionnelles ${ }^{37}$ qu'au niveau de la recherche d'une structure unique à " l'échelle idéale ${ }^{38}$. Ainsi, la complexité territoriale ${ }^{39}$ observée dans le Valenciennois n'apparaît pas nécessairement comme un obstacle si les acteurs institutionnels souhaitent favoriser la transversalité de leurs politiques respectives.

Néanmoins, le fonctionnement actuel des deux structures d'agglomération contribue à scinder le territoire en deux, et vient complexifier et instrumentaliser le rôle du SITURV qui doit alors composer en permanence avec les deux communautés. Jour après jour, les deux structures s'autonomisent davantage dans leur fonctionnement et les coopérations se raréfient. Présentée par certains comme une étape transitoire, il semble au contraire que cette structuration puisse perdurer tant que les clivages politiques resteront marqués.

37 Dans l'arrondissement, il semble que la recherche de cohérence entre les politiques de transport et d'urbanisme passe par la coordination des deux communautés d'agglomération, du syndicat mixte des transports, du conseil général et de la région (le PTU du Valenciennois comprend 11 gares du réseau ferré régional).

${ }^{38}$ Ce qui est décisif, selon Martin Vanier, à l'égard du sens des limites territoriales, ce n'est pas de « repousser pour coopérer dans de nouveaux périmètres (mythe de l'optimum dimensionnel), mais de considérer ces limites entre collectivités comme l'enjeu même des coopérations, en somme comme des membranes plutôt que comme des frontières »(Vanier, 2002).

${ }^{39}$ Voir sur ce sujet : Giraut F., Vanier M., 1999. Plaidoyer pour la complexité territoriale. in Gerbaux F. (dir.). Utopie pour le territoire : cohérence ou complexité ? éd. de l'Aube, p.143-172. 


\section{BIBLIOGRAPHIE}

○ Chaboche J., 2001. La diffusion spatiale de l'intercommunalité à fiscalité propre. Mappemonde $\mathrm{n}^{\circ} 63$, vol.3, pp.35-39.

- Dall'Aglio S., Petitet S., 2000. Territoire communal et solidarité territoriale, le cas de Villefranche-sur-Saône. L'Espace Géographique vol.2, pp. 170-183.

- Dechy F., 2001. Intercommunalité et Territoire, Étude du processus ayant conduit à la constitution de deux communautés d'agglomération au sein de l'arrondissement de Valenciennes. Mémoire d'IEP, Rennes, $82 \mathrm{p}$.

- Di Commo F., 2005. Le tramway en France: un symbole de la modernité urbaine. Pouvoir Locaux n ${ }^{\circ} 66$, pp. 84-86.

- Calame P., Talmant A., 1997. L’État au cour, le Mécano de la gouvernance. Ed.Desclée de Brouwer, Paris, $212 \mathrm{p}$.

- Frère S., Menerault Ph., Roussel I., 2000. PDU et dynamique des institutions à Lille et Valenciennes. Recherche Transports Sécurité, n69, pp.22-34.

- Frère S., 2001. L'intégration des préoccupations environnementales préconisées par la loi sur l'Air dans les Plans de Déplacements Urbains. Rapport final PRIMEQUAL / PREDIT, 101 p.

- Frère S., Richer C., 2004, Recomposition intercommunale et enjeux des transports publics en milieu urbain Le cas de l'arrondissement de Valenciennes, rapport de convention INRETS/APPA, PREDIT.

- Frère S., 2005, Concertation et décision dans les dispositifs de planification de la Loi sur l'Air (1996). Etudes dans le Nord Pas de Calais. Thèse de doctorat de science politique, Université de Lille2, 372 pages, soutenue le $1^{\text {er }}$ décembre 2005.

- Gallez C., 2005. Les transports urbains face à la structuration du pouvoir d'agglomération. Pouvoir Locaux n66, pp. 70-74.

- Gallez C., Menerault P. (dir), 2005. Recomposition intercommunale et enjeux des transports publics en milieu urbain. Rapport de convention Inrets-Ademe, 277 p.

- Gaxie D., 1997. Stratégie et institutions de l'intercommunalité. in CURAPP, l'intercommunalité, bilan et perspectives. PUF, pp. 25-50.

- Gonin P., 1996. Pays et développement local : l'état de la question dans la région Nord-Pasde-Calais. Hommes et Terres du Nord, n², pp.113-121.

- Le Saout R., Madoré F (dir). 2004. Les effets de l'intercommunalité. coll. Espace et territoires, Presses universitaires de Rennes, $224 \mathrm{p}$.

- Menerault P., 2005. Réforme territoriale et dynamique de l'intercommunalité dans les transports collectifs urbains : une approche diachronique. pp. 15-23. in Gallez C., Menerault P. (dir), 2005.

- Menerault P., 1993. Les effets territoriaux d'un outil de financement des transports publics : le versement-transport. Transports Urbains, ${ }^{\circ} 78$, pp. 21-24.

- Menerault P., 1995. Approche géo-institutionnelle des relations entre transports publics urbains et territoires à l'échelle du Nord-Pas-de-Calais. Hommes et Terres du Nord, $\mathrm{n}^{\circ} 3$, pp.161-166.

- Offner J.M., Menerault P., Sachet S., 2002. Les transports urbains, entre secteurs, réseaux et territoires. in Annuaire 2002 des collectivités locales, CNRS édition, Paris, pp. 169-184

- Richer C., 2005, Les transformations récentes de l'intercommunalité en matière de transport. pp. 25-49. in Gallez C., Menerault P. (dir), 2005.

- SEMURVAL, 1983. Un siècle de transports publics dans le Valenciennois. Valenciennes.

- Stackowiak S., 2001. Les difficultés de l'intercommunalité dans le Valenciennois. Mémoire de maîtrise, Université de Lille 1, UFR de géographie-aménagement, $126 \mathrm{p}$.

- Subra P., 1996. Le temps d'une reconversion, le Valenciennois (1965-1995). Presses Universitaires de Vincennes, Saint-Denis, 259 p.

- Subra P., 1993. Déménagement du territoire et démocratie: le Valenciennois (1960-1990). Hérodote $n^{\circ}$ 69-70, Paris, pp. 195-223

- Vanier M., 2004. Des grandes espérances aux effets sur l'arrangement territorial. in Le Saout R., Madoré F (dir). Les effets de l'intercommunalité. coll. Espace et territoires, Presses universitaires de Rennes, $224 \mathrm{p}$. 
- Vanier M., 2002. Les espaces du politique: trois réflexions pour sortir des limites du territoire. in Debarbieux B., Vanier M. (dir). Ces territorialités qui se dessinent. De L'aube, DATAR, pp. 75-89. 